\title{
COMMUNICATION
}

\section{Kyste foetal lingual : une localisation exceptionnelle}

\author{
Melka A, Pham Dang N, Picard M, Mondi JM, Barthélemy I \\ 1 - CHU Clermont-Ferrand, Service de Chirurgie Maxillo Faciale \\ 2 - Université d'Auvergne, Faculté de médecine \\ 3 - Université d'Auvergne, UMR Inserm U1107, Neuro-Dol, Trigeminal Pain and Migraine, Faculté de Chirurgie \\ dentaire
}

Mots clés : mucocèle, lingual, congénital

Introduction

Les mucocèles sont des lésions communes de la cavité orale, classées par types : extravasation ou rétention. À notre connaissance, la littérature rapporte 3 cas de mucocèles des glandes de Blandin-Nühn au niveau de la face ventrale de la langue. Cependant c'est le premier cas de mucocèle de la face ventrale de la langue avec un diagnostic ante-natal.

\section{Cas}

Nouveau-né de sexe masculin, né par voie basse spontanée à 38 semaines d'aménorrhée et 4 jours; sans antécédent familial d'anomalie congénitale. À $25 \mathrm{SA}$, une lésion kystique sublinguale de $25 \mathrm{~mm}$ a été découverte sur une échographie morphologique de routine, avasculaire, anéchogène à paroi fine, mobile à la déglutition, sans aucune autre anomalie morphologique. L'IRM à 30 SA retrouve un signal liquidien, homogène, hypersignal T2, hyposignal T1 et diffusion, d'allure stable : $26 \mathrm{~mm}$ de diamètre. Le kyste est sus mylo-hyoidien, développé aux dépens de la face ventrale de la langue. Pour pallier toute détresse respiratoire fœtale à la naissance, une ponction vacuatrice trans-amniotique est réalisée à $36 \mathrm{SA}$ avec succès sous contrôle échographique. En cas d'échec de cette ponction une équipe multidisciplinaire se serait alors tenue prête à inciser le kyste dès le dégagement de la tête. L'examen clinique du nouveau-né retrouve une langue tendue et violacée avec une masse souple, non inflammatoire, sur la face ventrale de la pointe de la langue, avec une ouverture pathologique de l'angle mandibulaire due à l'inocclusion labiale ; et une impossibilité totale d'alimentation. Des ponctions itératives sont alors réalisées. La cytologie retrouve un contenu muqueux sans argument pour un lymphangiome. Devant le volume du kyste malgré les ponctions, une marsupialisation première est réalisée à J2 sous anesthésie générale. Elle a permis un affaissement du kyste, un retrait de la SNG et un retour rapide à domicile. À l'âge de 5 mois, la résection chirurgicale de la lésion a été réalisée sous anesthésie générale. La dissection a révélé un kyste volumineux médian, et un petit kyste au niveau de la pointe de la langue. L'histologie a confirmé que la lésion médiane était un kyste mucoïde et un tératome à la pointe.

Discussion

La localisation linguale des mucocèles congénitales est exceptionnelle, posant le problème de son diagnostic différentiel avec les autres masses kystiques intra-linguales. La ponction lésionnelle et l'examen histologique permettent d'en faire la distinction car la présentation clinique est similaire. Ce cas pose également la question d'une marsupialisation ou d'une exérèse directe, devant le risque d'œdème et de trachéotomie chez le nouveau-né.

This is an Open Access article distributed under the terms of the Creative Commons Attribution License 4.0, which permits unrestricted use, distribution, and reproduction in any medium, provided the original work is properly cited. Article disponible sur le site nttp://www.stco-congres.org ou nttp://dx.dol.org/10.1051/stco/20166402025 